\title{
Aristotele, Eraclito e la forza irresistibile del thumos (22 B 85 DK)
}

\author{
Cristina Viano \\ cristina.viano@wanadoo.fr \\ Centre National de la Recherche Scientifique (CNRS), Paris, França
}

\begin{abstract}
Questo articolo presenta un quadro dei problemi che il frammento B85DK di Eraclito solleva e delle interpretazioni antiche e moderne che sono state suggerite. In particolare, è esaminata la testimonianza di Aristotele, la più antica e anche la più profonda e articolata. Una panoramica sui significati di thumos, punto centrale del frammento, mostra che per Aristotele questo concetto non si esaurisce nel pathos dell'ira. Il thumos è in primo luogo una dunamis, una facoltà dell'anima che rende possibile non solo il pathos, l'affezione passeggera dell'ira, ma anche il coraggio, l'impulsività, l'affetto, il senso della libertà e dell'autorità. Questa nozione aristotelica, così ricca e polimorfica da sottrarsi a una traduzione unica esaustiva, proviene in realtà dalla sistematizzazione di un materiale molto antico, omerico e presocratico, legato ai proverbi. Eraclito stesso ne offre già una formulazione che sintetizza due aspetti che Aristotele accoglie e sottolinea: la forza irresistibile e inarrestabile dello slancio impulsivo naturale, una volta che sia stato innescato, e l'estrema conseguenza a cui puo' portare, e cioè la perdita della vita stessa.
\end{abstract} parole chiave Eraclito; Aristotele; thumos; ira; pathos; dunamis; frammento B85DK

La versione ufficiale del frammento, B 85 DK di Eraclito, tratto da Plutarco, Coriolano, 22 è così formulata:

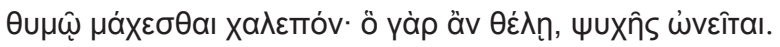

E' arduo combattere contro il thumos: cio' che vuole lo compra a prezzo della vita (o: dell'anima).

Ma la testimonianza più antica si trova in Aristotele, che lo cita ben tre volte, in una versione più sintetica, e in tre contesti morali, nell' Etica Recebido em 13 de agosto de 2013. Aceito em 27 de setembro de 2013. doispontos, Curitiba, São Carlos, vol. 10, n. 2, p.169-188, outubro, 2013 
Eudemia, nell' Etica Nicomachea e nella Politica, come una doxa notevole, autorevole e accettata, per illustrare il carattere irresistibile del thumos e la difficoltà che si incontra nel dominarlo.

Vorrei qui cominciare col presentare un quadro dei problemi che questo frammento solleva e delle interpretazioni di esso che sono state suggerite dagli antichi e dai moderni, e poi esaminare in dettaglio la testimonianza di Aristotele, da cui prendono il via tutte le altre e che è stata, a mio parere, considerata in maniera troppo riduttiva se non addirittura fraintesa. In realtà, l'interpretazione che Aristotele dà di questa sentenza eraclitea, è profonda e articolata, e costituisce un esempio luminoso della fecondità filosofica del pensiero eracliteo.

\section{Il frammento}

La traduzione di questa sentenza di Eraclito non va da sé e dipende direttamente dal senso che viene dato a thumos e a psuchê.

Per fare tre esempi: tra i più recenti: Fronterotta 2013 traduce: «E' arduo combattere contro la passione che ribolle nel cuore, perchè cio' che pretende lo compra a prezzo della vita»; Colli 1980, traduce «Contro la brama della passione è arduo combattere: qualsiasi cosa voglia, difatti, essa è disposta a pagarla con l'anima», mentre Conche 1986, che utilizza la citazione di Aristotele: "Il est difficile de combattre la colère, car elle l'emporte à prix de la vie».

Secondo Marcovich 1978, Eraclito avrebbe qui collegato due espressioni popolari, che sono del resto facilmente reperibili, anonime, nella letteratura greca. Per esempio, la difficoltà di combattere contro il thumos è espressa nelle Leggi di Platone («Il thumos, possesso connaturale, litigioso e difficile da dominare», IX, 863 B) e nella Medea di Euripide [«il thumos è più forte delle mie risoluzioni» (thumos de kreissôn tôn emôn bouleumatôn), 1079], mentre l'espressione «dare la vita (psuchê) in cambio di qualcosa» si puo' trovare nella Ciropedia di Senofonte, in cui un personaggio (Tigrane) dice che darebbe la vita (kan tês psuchês priaimên, III, 1,36) perchè sua moglie non sia mai schiava, o ancora nella Medea [«Per salvare i miei figli dall'esilio, darei la vita- (psuchês an allaixametha) e non solo il mio oro", 968]. 
Sembra del resto che per Eraclito sia una pratica frequente quella di fissare espressioni proverbiali della saggezza popolare in formule sintetiche e antitetiche. Basti pensare ai frammenti che evocano il mondo animale, come B9: «Gli asini preferirebbero la paglia all'oro» o B 13: «i porci godono più del fango che dell'acqua pura», immagine che troverà un'immensa fortuna sia nella letteratura pagana che in quella cristiana. Nel caso particolare del nostro frammento, le due espressioni congiunte sembrano addirittura costituire un proverbio.

Il problema fondamentale in B 85 è di comprendere cosa significhi thumos, e quale sia il suo rapporto con la psuchê (anima o vita).

\section{La dossografia}

Le testimonianze antiche che vengono dopo Aristotele non presentano conflitti apparenti e sembrano tutte concordare nell'identificare il thumos di Eraclito con l'ira, un impulso negativo da reprimere in ogni caso.

Plutarco cita tre volte B 85 nella stessa versione: secondo gli interpreti (cf. Fronterotta, 2013, p. 310) questa sarebbe una prova dell'affidabilità della sua fonte. Nella Vita di Coriolano (22, 3, testo DK $=\mathrm{a}^{1} \mathrm{C}^{2}$ Marcovich), Coriolano (personaggio collerico), recandosi da Tullo Attio in preda alla collera e rischiando la vita, illustra (marturian apelipe) col suo agire la massima eraclitea. Nel De cohibenda ira, $457 \mathrm{~d}$ ( $\mathrm{a}^{2} \mathrm{P}^{3}$ Marcovich), la stessa massima mostra lo sforzo che richiede resistere alla collera. Infine nell'Amatorius, $755 \mathrm{~d}$ ( $\mathrm{a}^{3} \mathrm{R}^{4} \mathrm{M}$.), leggiamo «è l'Amore che è difficile combattere, e non come diceva Eraclito, il thumos: ciò che vuole l'acquista anche al prezzo della vita, della ricchezza o della reputazione». Ritroviamo qui l'alternativa tra thumos e passione amorosa (chiamata qui un pathos dell'anima), ma il livello è quello dell'appropriazione della sentenza eraclitea da parte di Plutarco ai fini del discorso sulla forza irresistibile dell'amore. Alla luce delle due precedenti citazioni è probabile che Plutarco, anche qui, identifichi il thumos all'ira.

Nel cap. 21 del Protrettico di Giamblico (21, $8=$ c R M.) il simbolo pitagorico «non attizzare il fuoco col coltello» (= 58 c $6 \mathrm{DK})^{5}$, che invita a non contraddire un uomo "pieno di fuoco e di thumos", trova una testimonianza (martus tois lechtheisin Herakleitos) in Eraclito: «Lottare 
contro la collera è difficile, poichè quello che desidera veder accadere, lo acquista al prezzo dell'anima». Questa espressione che fa pensare alla vendetta proiettata nel futuro e altre come «dall'odio nasce l'amicizia, per l'estinzione del fuoco della collera» (puros thumikou), mostrano che anche qui il thumos è identificato alla collera che bisogna reprimere ${ }^{6}$.

Vi è ancora un passo di Filodemo (De ira, 27, 17= e M.) che condanna radicalmente l'ira (thumos) e che riecheggia B 85 senza però fare il nome di Eraclito: «L'ira...spinge a non risparmiare nemmeno le persone più care, con una cupidigia che compra ad ogni costo ciò che desidera» (met' epithumias pantos ônoumenês ha pothei). 'E stata fatta l'ipotesi ${ }^{7}$ che qui Filodemo, seguendo Aristotele, fraintese il thumos eracliteo, considerandolo equivalente all'ira. Mi pare più verosimile l'ipotesi di Marcovich, che Filodemo abbia qui attinto direttamente a un'espressione proverbiale.

Ora, l'identificazione da parte di Aristotele del thumos eracliteo con l'ira è considerata dalla totalità degli interpreti come un dato di fatto. Secondo una buona parte dei critici, e in particolare, come vedremo, i partigiani del «desiderio del cuore», Aristotele avrebbe frainteso Eraclito, o voluto ridurre, come al solito, il pensiero eracliteo nel letto di Procuste dei propri schemi concettuali.

Anche se la collera appare spesso per Aristotele come il paradigma delle passioni, tuttavia questa identificazione thumos-collera, a un esame più approfondito dei testi aristotelici non risulta poi così evidente e in ogni caso non così restrittiva. Non ci sarebbe da discutere se per Aristotele l'accezione di collera esaurisse tutti i significati di thumos.

\section{Le interpretazioni moderne}

Le interpretazioni moderne possono essere raggruppate in tre principali tendenze. Una identifica thumos con la passione, con il desiderio sensuale e erotico, l'epithumia platonica e aristotelica. Su questa linea troviamo, per esempio, Cl. Ramnoux 1968 («énergie érotique») e G. Colli, 1980 («brama della passione»). Altri identificano invece il thumos con l'ira, l'aggressività, l'impulso bellicoso, come Kahn 1979 ("passion», ma specifica poi che si tratta della collera), Schofield-Kirk-Raven $1983^{2}$, Mansfeld 1992 («anger»), Conche, 1986 («colère», principio del polemos), Mouraviev 1991 («ire»). 
Queste due interpretazioni pongono un'alternativa, che Ramnoux 1968, esprime in questi termini: o la formula eraclitea è "eroica» e elogiativa (l'ardore bellicoso irresistibile dell'eroe che sacrifica la propria vita) ${ }^{8}$, oppure la formula è «ascetica» e prescrittiva (bisogna resistere all'impulso sensuale-erotico, secondo Ramnoux, poichè consuma la riserva di vitalità che ciascun uomo possiede) $)^{9}$.

Ma la tendenza maggiore, più prudente, propone di tradurre thumos con un termine più indeterminato: «cuore», come ardore o foga vitale (cf. «élan du cœur», Bollack-Wismann 1972), «impetuosità, impulso passionale» (Mondolfo 1968², mentre Zeller 1892 identificava radicalmente -e negativamente- il thumos all'hubris, come una colpa da reprimere), il «proprio impulso emotivo» (CAPIZZI 1979), il «cuore» (HERZ, DIELS 1909), o ancora «desiderio del cuore» (BURNET 1930, MARCOVICH 1978), intendendo cuore nel senso omerico di organo indipendente, di centro del desiderio, di sede delle passioni e delle emozioni, comme collera e epithumia (cf. Diano 1980, «il proprio animo»; Robinson 1991, «passion»).

Le soluzioni proposte si possono dunque riassumere in: passione sensuale, ira e «cuore», come impulso emotivo e indifferenziato.

\section{Aristotele}

Veniamo ora alla testimonianza aristotelica, e esaminiamola in dettaglio. Vorrei ricordare che è la fonte più antica che conosciamo e che ci trasmette una versione più sintetica di B 85 rispetto a quella di Plutarco, omettendo ô yà ồv $\theta \dot{c} \lambda$ n ( «cio' che vuole»), in tre contesti diversi: nell' Etica Eudemia, nell' Etica Nicomachea e nella Politica. Come vedremo, il contesto delle Etiche e quello della Politica offrono due interpretazioni diverse della sentenza eraclitea ${ }^{10}$.

\subsection{Eth. Eud., II 7, 1223 b 18-28 (= b ${ }^{1}$ R M.): thumos e akrasia}

Lo stesso discorso vale per il thumos: infatti sembra esservi mancanza di controllo e controllo del thumos, come del desiderio irrazionale; e cio' che contrasta il thumos è penoso e la sua repressione forzata, di modo che, se cio' che è represso è involontario, cio' che è conforme al thumos, sarà totalmente volontario. Anche Eraclito sembra dire, fissando 
lo sguardo sulla forza del thumos, che è penoso opporvisi. 'E' difficile, dice, combattere contro il thumos: si paga a prezzo dell'anima'. Ma se è impossibile che la stessa persona faccia, al tempo stesso, volontariamente e involontariamente, la stessa cosa, sotto lo stesso rispetto della cosa, sarà più volontario l'[atto] conforme al desiderio volontario, che l'atto conforme al desiderio irrazionale e al thumos. Ecco la prova: facciamo molte cose volontarie senza collera e senza desiderio irrazionale ${ }^{11}$.

Il passo è complesso. Il contesto è la discussione sulla definizione di cosa sia il volontario (hechôn). Aristotele prende in esame il desiderio, nelle sue tre accezioni di desiderio volontario, di desiderio impulsivo e di desiderio irrazionale (boulêsis, thumos, epithumia, cf. 1223 a 26). Qui il discorso ha come oggetto la mancanza di controllo (akrasia) e il controllo (enkrateia) del thumos e Aristotele collega la repressione del desiderio con l'azione volontaria o involontaria. La sentenza di Eraclito è introdotta per esprimere quanto sia penoso contrastare il thumos. In questo senso è inteso il nostro frammento. Si noterà che Aristotele non assimila direttamente la sofferenza che implica la repressione del thumos con la difficoltà che si prova a combatterlo, ma dice: «sembra che anche Eraclito dica...che è penoso reprimerla». Si noterà inoltre che in tutto il passo thumos per Aristotele non significa propriamente collera ma quella forma particolare di desiderio che è l'impetuosità.

Il termine "collera» (orgê) compare solo alla fine, nella "prova» che il volontario non si identifica nè al thumos nè all'epithumia: «facciamo molte cose volontarie senza collera e senza desiderio irrazionale». Penso che sia questa frase che abbia portato gli interpreti a pensare che Aristotele intendesse il thumos eracliteo come collera.

\section{2. Eth. Nic. II 2, 1105 a 7-13 (= $\mathrm{b}^{3}$ R M.): thumos e piacere}

Inoltre è più difficile combattere contro il piacere che con il thumos, come dice Eraclito, e l'arte e la virtù sorgono sempre riguardo alle cose più difficili, dato che anche il successo è migliore in questi casi. Infatti in quest'ambito il bene è di natura ancora superiore. Di conseguenza, anche per questo motivo, tutta la trattazione ha per oggetto i piaceri e i dolori, sia rispetto alla virtù che rispetto alla politica, dato che colui che si comporta bene in questi campi è buono, chi si comporta male è cattivo ${ }^{12}$. 
Aristotele qui sviluppa la sentenza di Eraclito, aggiungendo che è più difficile combattere contro il piacere che contro il thumos. Anche questa volta la doxa eraclitea, limitata al thumos, è introdotta positivamente in un contesto relativo al rapporto tra azioni e piacere, per mostrarne la forza come motore dell'azione («è a causa del piacere che compiamo le cose cattive ed è a causa del dolore che ci asteniamo dalle cose moralmente belle», $1104 \mathrm{~b}$ 9). La maggior parte dei critici esclude che tutta la frase sia un'altra sentenza di Eraclito, in cui egli avrebbe paragonato il thumos al piacere ${ }^{13}$.

Tra le tre cose muovono le scelte (eis aireseis), il bello, l'utile e il piacevole (hedu). Quest'ultimo è comune agli uomini e agli animali ed a esso possono ricondursi gli altri due. Esso si accresce fin dall'infanzia ed è quindi difficile sbarazzarsi di questo pathos che è strettamente mescolato con la vita stessa (1105 a 3).Va quindi educato, infatti: «come segno delle disposizioni morali dobbiamo assumere il piacere e il dolore per le opere» (1104 b 3).

Il piacere è definito in Eth. Nic. X 4, 1174 b 32 come un fine che perfeziona l'atto e viene ad aggiungersi (epiginomenon) al pari della bellezza, a chi è nel fiore dell'età.

Si tratta di qualcosa di profondamente connaturato alla natura umana, di insopprimibile e di indisgiungibile dal fine, sia esso morale o malvagio, a cui l'azione tende.

L'Anonimo in Eth. Nic. (II-IV 129, 1) commenta:

Dice [Eraclito] che è più difficile combattere contro il piacere che contro il thumos (nella traduzione latina di Grossatesta si trova ira), poichè anche il thumos è più difficile da vincere (dusmachôteros), qualora appaia contemporeanamente (hama) al piacere, quel thumos, che come dice Eraclito, molto più dolce del miele stillato, cresce nei petti degli uomini come fumo (=Iliade, XVIII,109-110).

Paul Moraux ${ }^{14}$ considera questa attribuzione come un errore ${ }^{15}$, non del Commentatore $^{16} \mathrm{ma}$ del copista (o del compilatore). Infatti questa ultima frase non è di Eraclito, ma sono versi dell' Iliade, citati anche in Rhet. I 11, 1370b 11 e II, 2, 1378b 5, a proposito del piacere che la collera puo' implicare. Si tratta del piacere che deriva dalla speranza di vendetta come anticipazione di cio' che si desidera, ovvero rendere la pariglia e ristabilire così la propria integrità alterata da un atto di disprezzo. La collera di per sé, quando insorge, è un'emozione accompagnata dal dolore. 
Probabilmente il piacere difficile da combattere di cui parla Aristotele in questo contesto non è il piacere della vendetta, ma il piacere sensibile, a cui tende il desiderio irrazionale (epithumia).

In ogni caso, l'accostamento tra le parole di Eraclito e questi versi di Omero merita di essere sottolineato perchè stabilisce un rapporto diretto tra il thumos omerico e quello eracliteo.

\section{3. Polit.V, 11,1315 a $24-31$ (= $\mathrm{b}^{2}$ R M.): thumos e vendetta}

Fra coloro che attentano alla vita del tiranno, sono più da temere e esigono da parte sua la più grande vigilanza, tutti coloro che non deliberano di conservare la vita pur di uccidere. Per questa ragione, il tiranno deve guardarsi innanzitutto da coloro che si credono oltraggiati nella propria persona o in quella di qualcuno che sta loro a cuore. Infatti, coloro che attaccano per il thumos non si risparmiano, come dice Eraclito, affermando che è difficile lottare contro il thumos: infatti, si compra a prezzo dell'anima ${ }^{17}$.

Il contesto porta sui modi di preservare la tirannide. Anche qui la sentenza di Eraclito è introdotta per illustrare la posizione aristotelica. Aristotele fornisce un'interpretazione anche della seconda parte della sentenza eraclitea: pagare a prezzo dell'anima significa non risparmiarsi la vita. Dia thumon mostra che il thumos è la causa dell'azione.

Questo tipo di thumos dei tirannicidi ha a che fare con la collera poiché, come vedremo, la collera è definita da Aristotele come il desiderio di vendetta per una mancanza di rispetto (oligoria) nei nostri confronti o di qualcuno dei nostri. Ora, l'oltraggio (hubris) è una forma particolare di mancanza di rispetto che consiste in atti o parole che possono fare provare vergogna a qualcuno, senza nessun altro fine che questo risultato o il piacere di farlo (Rhet. II, 2, 1378b 24).

Come osserva acutamente Mansfeld 1992 (p. 14 ss.), Aristotele fornisce qui una intepretazione della sentenza eraclitea diversa rispetto ai due passi precedenti. Qui infatti, si riferisce esplicitamente ai tirannicidi che non tengono conto della propria vita. Come nei passi precedenti, psuchê e thumos appartengono alla stessa persona ma il conflitto (machesthai) non avviene all'interno del soggetto, con la propria collera, ma all'esterno con la collera di un altro. Ora, per Mansfeld è proprio l'interpretazione morale 
delle Etiche, riferita a un conflitto interiore, tra emozione e ragione, l'interpretazione più filosoficamente notevole e "profonda» della sentenza eraclitea $^{18}$. Secondo Mansfeld (p. 18), il thumos è une manifestazione della psuchê e quando la collera prende il sopravvento, la razionalità diventa più debole perchè parte della forza vitale è stata convertita in collera. In altri termini, la collera prende possesso dell'anima e porta l'individuo alla perdizione. Thumos e psuchê esprimono due facce della stessa medaglia.

Come si puo' osservare, questi tre contesti aristotelici di B 85, non trattano propriamente della collera, anche se nel primo e nel terzo questa appare come un caso specifico di thumos. Cerchiamo ora di capire cosa fosse precisamente il thumos per Aristotele tracciando una breve panoramica delle principali accezioni di questo termine nel corpus aristotelico.

\subsection{L'impulsività (emportement)}

In Ethica Nic. VII 7, 1149 a 25-b3, il thumos è descritto nei termini dell'impulsività, della precipitazione:

Il thumos sembra infatti ascoltare in qualcosa la ragione, ma ascoltarla di traverso, come i servitori precipitosi, i quali, prima di aver ascoltato tutto quello che vien detto loro, schizzano via, e poi sbagliano l'esecuzione dell'ordine; e come i cani che, prima di aver osservato se è un amico, appena soltanto si bussa alla porta, abbaiano. Così il thumos, che per il calore e la precipitazione della sua natura avendo pur ascoltato la ragione, ma non sentendone i dettami muove alla vendetta. Infatti la ragione o l'immaginazione mostrano che ci sono stati commessi un oltraggio e un'offesa, e il thumos, come se un ragionamento gli avesse fatto concludere che si deve muovere guerra contro chi ha compiuto un tale atto, subito pertanto si infuria. La brama per contro, se soltanto la ragione e la sensazione dicono: è piacevole, muove verso il godimento. Di conseguenza, il thumos segue in qualche modo la ragione, la brama no. L'intemperanza relativa a quest'ultima è dunque più turpe. Infatti l'intemperante in materia di thumos è in un certo senso vinto dalla ragione, l'altro invece è vinto dalla brama e non dalla ragione ${ }^{19}$.

La mancanza di controllo del thumos è considerata meno vergognosa che nei desideri sensuali. Le ragioni sono essenzialmente quattro. In primo luogo, l'impetuoso (thumodes) ascolta la ragione, anche se la ascolta male. 
Poi, il thumos fa parte dei desideri naturali (phusikai orexeis), è radicato nella natura (cf. 1149 b 10: sunghenes), è più naturale della brama che mira all'eccesso o a ciò che non è necessario. Inoltre, l'impetuoso non usa macchinazioni ma agisce allo scoperto. Infine, chi agisce per collera, agisce provando dolore, mentre chi oltraggia lo fa con piacere.

Qui è difficile distinguere collera da thumos. Si direbbe pero' che il thumos sia un impulso radicato, connaturato, fisico e costante, mentre la collera sia uno stato passeggero che risulta dall'orientamento verso la vendetta. Da questo passo appare anche che lo slancio della brama, del desiderio irrazionale (epithumia) verso il piacere è senza mediazioni. Forse è per questo motivo che è più difficile resistere?

\subsection{Il «cuore»}

In Pol.VII, 7, 1327 b 39, leggiamo:

Cio' che secondo alcuni deve caratterizzare i Guardiani, e cioè sapersi mostrare benevoli verso coloro che conoscono, e senza pietà verso coloro che non conoscono, è in realtà il thumos (Tricot: «cuore ») che produce la capacità d'amare. E' questa infatti la facoltà dell'anima per mezzo della quale noi amiamo. Ne è prova il fatto che il thumos si solleva più contro gli amici e gli intimi che contro gli sconosciuti, quando si crede disdegnato da essi[...]. (1328 a 7) Il senso dell'autorità e quello della libertà derivano per tutti gli uomini da questa facoltà, poichè il thumos è qualcosa di imperioso e di indomabile ${ }^{20}$.

\subsection{Il thumos e il coraggio}

Molto vicino al thumos è il coraggio. Aristotele però distingue chiaramente questi due concetti. In Eth. Eud. III, 1, 1229 a 20, leggiamo:

Un altro tipo di coraggio è dovuto a un sentimento irrazionale, come l'amore $\mathrm{o}$ il thumos; infatti un uomo in amore è più temerario che pavido e affronta numerosi pericoli [...]. Lo stesso vale per l'azione della collera e del thumos, poichè il thumos getta qualcuno fuori di sè. E' per questo motivo che i cinghiali sembrano coraggiosi, ma non lo sono, poichè, quando sono fuori di sè, hanno un comportamento ma, se non lo sono, sono imprevedibili, come i temerari. Tuttavia il coraggio del thumos è il più naturale: infatti il thumos è qualcosa di invincibile $e^{21}$ 
E ancora, in Eth. Nic. III, 11, 1116 b 23-1117 a 9:

Riconducono anche il thumos al coraggio. Infatti sono considerati coraggiosi anche coloro che agiscono per thumos, come le belve, le quali si scagliano su chi le ha ferite, perchè anche i coraggiosi sono impetuosi. Infatti il thumos è sommamente ardito verso i pericoli, donde Omero dice: «Infuse forza al suo impeto» e «destò il furore e l'impeto» e ancora: «L'aspro furore salì alle narici», ed inoltre: «Ribollì il sangue». Infatti tali espressioni sembrano significare tutte il risveglio del thumos e lo slancio. I coraggiosi agiscono dunque spinti dal bello e il thumos collabora con loro. Le bestie invece agiscono sotto la spinta del dolore: infatti agiscono per il fatto di essere ferite o di avere paura. [...] (1117 a 4) Ma la forma del coraggio che causa il thumos sembra essere la più naturale e, se ne assume inoltre l'intenzione e il fine è autentico coraggio. Pertanto anche gli uomini, quando sono irati, provano dolore e, vendicandosi, provano piacere. Ma coloro che combattono per queste ragioni sono battaglieri e non coraggiosi, non lo fanno per il bello nè perchè lo prescrive la ragione, ma spinti dalla passione. Però hanno qualcosa del coraggio ${ }^{22}$.

Cio' che distingue il coraggio dal thumos sono la causa e il fine, e ciò̀ la ragione e il bello. Tuttavia, anche se non vale l'inverso, tutti i coraggiosi sono impetuosi (thumoedeis) e il thumos, "collabora» col coraggio, addirittura "se ne assume inoltre l'intenzione e il fine » puo' diventare autentico coraggio.

Colpisce in questi passi il carattere attivo e dinamico del thumos in atto che "getta fuori di sè», o meglio "fuori dalla ragione " (ekstatikon, sottinteso tou logou). Le espressioni omeriche indicherebbero inoltre " il risveglio dell'impetuosità e lo slancio». Il risveglio fa pensare a un passaggio dalla potenza all'atto.

Il thumos appare dunque qui come una forza naturale indefinita, «estatica», che puo' essere circoscritta e trasformata in coraggio.

\subsection{Il thumos e la collera}

Come abbiamo visto, è difficile distinguere i due concetti, soprattutto nei contesti dell'etica e della retorica dove spesso sono associati o addirittura interscambiabili. In Rhet. I 10, 1368 b 38, thumos e ira rientrano entrambi, senza distinzione, nei desideri (orexeis) irrazionali che provocano le azioni 
volontarie. In 1369 b 12, i due termini sono uniti da un kai, che pottrebbe essere epesegetico o indicare che l'ira è la manifestazione paradigmatica del thumos: «Per impetuosità e per ira (dia thumon de kai orgên) si compiono le azioni di vendetta [...]. Cosa sia l'ira diventerà evidente quando tratteremo delle passioni (pathê)».

In Rhet. II, 2, 1378 a 30, abbiamo la celebre definizione della collera:

Definiamo dunque la collera come il desiderio, accompagnato da dolore, di vendetta manifesta, causato da cio' che appare come una mancanza di riguardo rivolta noi stessi o ai nostri, da parte di chi non ne ha il diritto. Se dunque è questo la collera, ne consegue necessariamente che colui che si mette in collera, si metta sempre in collera contro qualcuno di determinato, contro Cleone, per esempio, e non contro l'uomo in generale, e che abbia fatto o stia per fare qualcosa a noi stessi o qualcuno dei nostri, e che ogni collera sia seguita da un certo piacere che deriva dalla speranza di vendicarsi ${ }^{23}$.

Dice Aristotele che a ogni ira consegue (o meglio: «implica», il verbo epesthai indica qui una conseguenza logica e non temporale) un piacere proveniente dalla speranza di vendicarsi. E' infatti piacevole pensare di ottenere cio' a cui si aspira, "percio' è stato detto bene a proposito del thumos: 'molto più dolce del miele stillato, cresce nel petto degli uomini'» (Iliade, XVIII, 109-110).

Da questi passi, l'ira si caratterizza come pathos e appare come una forma di thumos circostanziato e rivolto contro qualcuno di ben preciso.

In De anima, I, 1, 403 a 17, abbiamo le due definizioni, dialettica e fisica della collera:

Sembra che tutte le affezioni dell'anima siano date con il corpo: il thumos, la dolcezza, il timore, la pietà, l'audacia, e ancora la gioia e l'amare e l'odiare [...].

Stando così le cose, è chiaro che le passioni sono forme calate nella materia e di questo, conseguentemente, si deve tener conto nelle definizioni: la collera, per esempio, è un movimento di tale corpo o di tale parte o di tale facoltà prodotto da tale causa per tale fine. Perciò spetta al fisico l'investigazione dell'anima nella sua interezza o nel particolare aspetto descritto. Il fisico e il dialettico definirebbero così in maniera differente ciascuna di queste affezioni, cos'è, ad esempio, la 
collera. Per il secondo è il desiderio di restituire l'offesa o qualcosa di simile; per il primo è il ribollire del sangue o del calore che sta intorno al cuore ${ }^{24}$.

La collera, come gli altri pathe, appare come "un movimento di tale corpo o di tale parte o di tale facoltà » dell'anima. La collera è un pathos molto preciso, non è uno slancio cieco e indeterminato. Qui emerge una distinzione importante: la collera è un pathos collegato a una dunamis, una facoltà dell'anima che lo rende possibile, evidentemente questa facoltà è quella desiderativa, l'orektikon e più precisamente il thumikon, ovvero il thumos. Nella collera, come nell'impetuosità e nel coraggio, il thumos sarebbe in atto.

\subsection{Gli effetti fisici dei thumoi}

Rimane da evocare ancora l'aspetto fisiologico dei fenomeni legati al thumos. Come abbiamo visto nella definizione "fisica» della collera del De anima, questo pathos è legato al calore. Leggiamo in De part. an. II, 3, 650 b 35:

Gli animali invece che hanno fibre abbondanti per numero e spessore, sono di natura più terrosa, di temperamento impetuoso e facili a lasciarsi trasportare dal thumos. Il thumos produce calore, e i corpi solidi, una volta riscaldati, emettono più calore dei fluidi: ora le fibre sono un elemento solido e terroso, sicché si comportano nel sangue come fonti di calore e, nei momenti di collera, ne producono l'ebollizione perciò i tori e i cinghiali si lasciano facilmente trasportare dal thumos: il loro sangue è più fibroso, e quello del toro presenta in assoluto la coagulazione più rapida ${ }^{25}$.

Da un punto di vista fisiologico il fenomeno si manifesta in un solo modo: è impossibile distinguere gli attacchi di collera, gli slanci emotivi e affettivi, le emozioni durante le azioni coraggiose (cf. Probl. X, 898 a 60 e XXVIII,3, 947 b 13). Si tratta in ogni caso di produzione di calore, di ebollizione del sangue, del movimento di una parte del corpo che ribolle e tende all'esterno. Il thumos si manifesterebbe in un particolare tipo di fibre che funzionano nel sangue come fonti di calore e lo portano a ebollizione.

A questo punto sorge spontanea la questione sul rapporto del thumos e delle sue manifestazioni fisiche di calore con la dottrina dell'anima-fuoco ${ }^{26}$ di Eraclito o più precisamente l'anima-anathumiasis (esalazione secca) di 
cui Aristotele ci da' notizia in De an. I, 2, definendola come un principio analogo al principio cosmologico (il fuoco). Questo porterebbe poi alla questione dell'influenza di Eraclito sulla dottrina psicologica di Aristotele stesso, sulla simpatia di Aristotele per la dottrina dell'anima-fuoco, in particolare nel De iuventute, dove si parla di anima «infiammata» ${ }^{27}$. Bisogna pero' riservare quest'analisi a un altro momento perchè non si puo' esaurire in poche parole. Quello che ho voluto mostrare in questa sede è l'ampiezza e l'articolazione del concetto di thumos per Aristotele lettore di Eraclito.

\section{Conclusione: Eraclito tra Omero e Aristotele}

Come risulta da questa panoramica sui significati di thumos è chiaro che per Aristotele questo concetto non si esaurisce nel pathos dell'ira. Il thumos è in primo luogo una dunamis, una facoltà dell'anima che rende possibile non solo il pathos, l'affezione passeggera dell'ira, ma anche il coraggio, l'impulsività, l'affetto, il senso della libertà e dell'autorità. Come pathos, il thumos appare come una sorta di slancio violento e rapido, un trasporto «fuori di sé», fuori dalla propria ragione (ekstatikon, in francese thumos viene tradotto spesso con «emportement»), un impulso irresistibile, come il caso dei servi precipitosi che corrono via ancor prima che il padrone abbia finito di dare gli ordini. Se dominato dell'enkrateia e orientato puo' essere concausa del coraggio, infatti esiste un coraggio del thumos, se vi è, al contrario, akrasia (debolezza del volere) questo slancio «fuori di sé» non si arresta nemmeno davanti alla morte. Direi che il termine più appropriato per definire il pathos in atto del thumos che non è la collera potrebbe essere 'spinta emotiva', qualcosa di simile a una scarica di adrenalina nel sangue, un fenomeno emotivo e fisiologico al tempo stesso.

Se il thumos non si esaurisce nell'ira per Aristotele, si puo' pensare ancor più a ragione, che sia così anche per Eraclito, che ha alle spalle tutta la tradizione omerica. E nulla costringe a pensare che Aristotele riducesse il thumos di Eraclito all'ira tout court. La funzione dell'affermazione di Eraclito è infatti qui positiva e viene utilizzata da Aristotele per esprimere la spinta emotiva irresisistibile generata da una funzione connaturata all'anima umana. 
Ma Aristotele non la discute nel senso moderno della restituzione del suo messaggio originario. Si puo' pensare piuttosto che usi questa espressione come un endoxon, nel suo senso più ampio e condiviso, e cioè non tanto nel senso corrente e tecnico di opinione notevole del passato da vagliare e criticare prima di inserirla nella sua argomentazione, ma piuttosto come una doxa d'appoggio particolarmente adatta a sintetizzare il suo pensiero ${ }^{28}$.

La difficoltà a combattere contro il piacere è un'aggiunta di Aristotele alla sentenza di Eraclito e mi pare che Aristotele non ne faccia mistero. Infatti non aveva nessun motivo di stravolgere una frase di Eraclito per fargli dire il suo pensiero. Sappiamo che Aristotele adatta più o meno abusivamente il pensiero dei Presocratici ai propri schemi concettuali, ma non aveva bisogno di alterarne le sentenze. In questo caso, il thumos sarebbe distinto dal desiderio (nel senso aristotelico di epithumia). Aristotele aveva in mente il paragone tra la sensazione del piacere e il desiderio impulsivo che la ragione ha difficoltà a reprimere. Quello che importa è che Aristotele attribuisce già a Eraclito la constatazione della forza di questi fenomeni psichici.

Quindi Aristotele avrebbe potuto intendere il frammento di Eraclito in questo modo: è difficile dominare la facoltà emotiva, una volta in atto puo' costare la vita (puo' essere sia l'ira che mira alla vendetta, sia l'impeto cieco dei barbari che non vedono i pericoli). L'opposizione tra le due parti dellla sentenza eraclitea sarebbe stata vista da Aristotele come l'opposizione tra la difficoltà dell'enkrateia e le conseguenze estreme a cui porta l' akrasia del thumos.

Ora, da un punto di vista metodologico, la citazione di questo frammento da parte di Aristotele è a mio parere un esempio magistrale dell'interesse e della fecondità filosofica che puo' avere la contestualizzazione di un frammento presocratico nell'opera aristotelica ${ }^{29}$.

In realtà, Aristotele non ci dice e non intende dire niente che ci permetta di capire cosa volesse dire Eraclito in questa sentenza. Ma ci fornisce delle preziose informazioni, sulle fonti, sul materiale e sul metodo della costruzione della sua dottrina morale del desiderio e delle passioni, e in particolare della nozione di thumos. Aristotele ci mostra infatti che questa nozione così ricca e polimorfica da sottrarsi a una traduzione unica esaustiva, proviene dalla sistematizzazione di un materiale molto antico, 
omerico e presocratico, legato ai proverbi, di cui Eraclito stesso offre una già una formulazione che sintetizza due aspetti che Aristotele accoglie e sottolinea: la forza irresistibile e inarrestabile dello slancio impulsivo naturale $^{30}$, una volta che sia stato innescato, e l'estrema conseguenza a cui puo' portare, e cioè la perdita della vita stessa. E' immagine dei barbari che, in preda alla loro temeriarietà naturale, si gettano contro i flutti in tempesta senza badare al rischio della propria vita. E' proprio su questo aspetto irresistibile e indomabile del desiderio, (impulsivo ma anche sensuale), che Aristotele fonda la sua teoria della virtù. Una teoria che, a differenza di quella platonica e poi cristiana, non consiste nella repressione ascetica degli impulsi naturali, ma nell'assecondarli e educarli alla giusta misura tra gli eccessi, fonti di distruzione.

${ }^{1}$ Queste riflessioni sono state presentate nell'estate del 2013 a Porto Alegre, Universidade Federal do Rio Grande do Soul, e all'Universitade de São Paulo (USP). Vorrei ringraziare tutti gli amici, colleghi e studenti che hanno discusso con me in queste due occasioni, e in particolare: Juliana Aggio,Vivianne de Castilho Moreira, Rodrigo Guerizoli, Christoph Horn, Jean-Louis Hudry, Maria Liatsi, Carlo Natali, Eduardo Wolf, Inara Zanuzzi, Raphael Zillig, Marco Zingano.

$2=$ Citazione.

$3=$ Parafrasi.

${ }^{4}=$ Reminescenza.

${ }^{5}$ La stessa associazione di questo symbolon pitagorico con B 85 si trova nel Commento al Filebo platonico di Marsilio Ficino (In Plat. II, 1228, ed. Basileae, 1576=f R M.): « E se temi l'ostinazione di quel Filebo, congedalo e non discutere con lui, infatti come dice il proverbio: 'Non bisogna smuovere un male che giace tranquillo' a cui è simile il simbolo di Pitagora:'non attizzare il fuoco col coltello' e quello di Eraclito: 'è difficile combattere contro l'ira, infatti, quando è in corsa, si paga con la vita' (Durum est adversus iram pugnare, nam cum in impetu est, vita emitur)». Cf. Phileb; 15 c: «...quanto a Filebo, forse è meglio che tu non smuova cio' che giace tranquillo, interrogandolo ora».

${ }^{6}$ Cf. anche Porph. V. Pyth. $42 \mathrm{~s}:$ «'Non attizzare il fuoco col coltello'; vale a dire non eccitare con discorsi aspri chi è gonfio di collera».

${ }^{7}$ Cf. Filodemo, L'ira, ed., trad. e commento di Indelli 1988, p. 196. Per una analisi recente di quest'opera di Filodemo, si veda Tsouna 2008.

${ }^{8}$ Cf. i frammenti B 25 («Maggiori destini di morte ottengono infatti maggiori ricompense») e B29 («Rispetto a tutte le altre, una sola cosa preferiscono i migliori: la gloria eterna rispetto 
alle cose caduche; i più invece pensano solo a saziarsi come bestie») che esaltano la morte dell'eroe.

${ }^{9}$ Sono spesso evocati i frammenti B 36 («Per le anime è morte diventare acqua, e per l'acqua è morte diventare terra, ma dalla terra nasce l'acqua e dall'acqua nasce l'anima»), B 117( «L'uomo, quando è ebbro, è condotto barcollante da un fanciullo imberbe, senza comprendere dove va, dal momento che la sua anima è umida») e B 118 («Secco splendore è l'anima più saggia, e migliore, o piuttosto: l'anima secca è la più saggia e la migliore»), che collegano le variazioni elementari e fisiologiche dell'anima alla morte e al comportamento etico.

${ }^{10}$ Cf. Mansfeld 1992, p. 14.

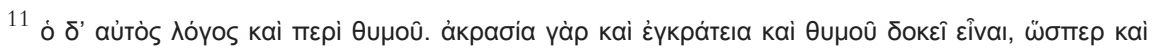

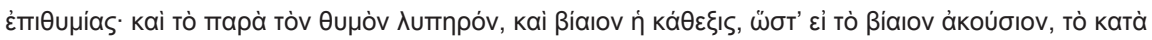

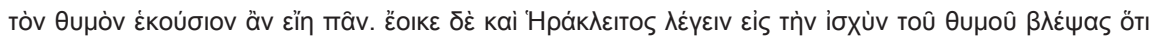

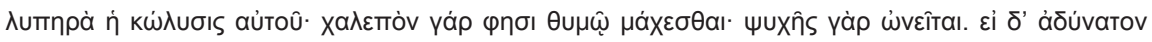

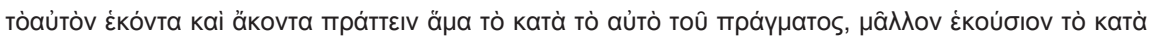

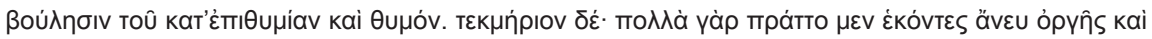

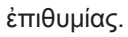

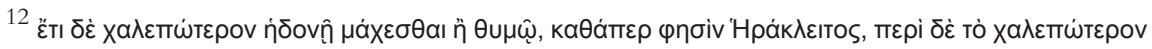

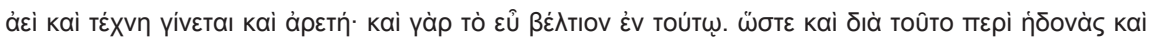

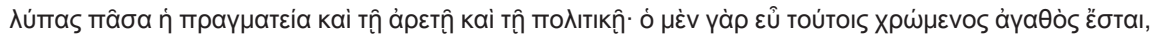

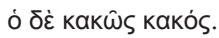

13 Cf. Fronterotta 2013, p. 310.

${ }^{14}$ Moraux 1973, II, 329 n.146. Cf. Mercken 1980, p. 407-422.

${ }^{15}$ Tra l'altro, in Eth. Eud.VII 1, 1235a 251, il verso 107 è messo in bocca a Eraclito come oggetto di critica: «Ed Eraclito rimprovera il poeta che scrisse: 'Così si estinguesse la discordia tra gli dei e gli uomini!'. Giacchè non ci sarebbe accordo musicale se non ci fossero l'acuto e il grave, nè ci sarebbero animali senza femmina e maschio che son contrari» (= 22 A 22 DK). Potrebbe essere questa la causa dell'errore? O forse Eraclito avrebbe commentato anche questi versi dell'IIliade?

${ }^{16}$ Sull'autore degli Scholia al secondo libro dell'Eth. Nic, cf. Moraux 1973; ib. p.2 94-332 e Mercken 1980, art. cit., p. 422. Questo personaggio viene identificato con Adrasto di Afrodisia (prima metà del II s. d. C.) e considerato come molto vicino allo spirito e alla lettera del testo aristotelico.

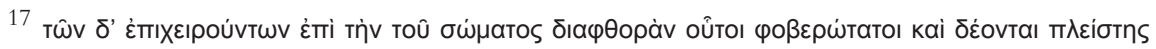

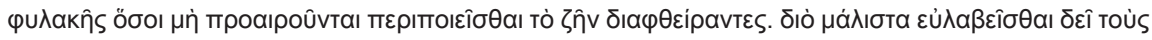

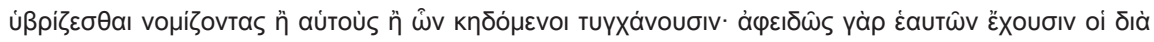

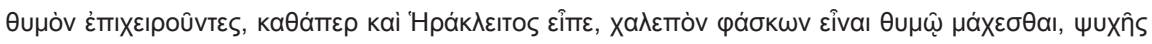

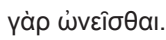

${ }^{18}$ Mansfeld 1992, p. 16 distingue tre interpretazioni possibili: (1) «superficiale»I (Politica): combattere contro qualcuno che è in collera è difficile perchè costui è pronto a rischiare la vita; (2) «superficiale " II (mai proposta prima): combattere quando si è in collera è pericoloso perchè cio' porta a rischiare la vita; (3) «profond» e moderna (Eth. Eud. e Eth. Nic.): si riferisce a un 
conflitto interiore: combattere la collera è difficile perchè paga a prezzo dell'anima cio' che desidera.

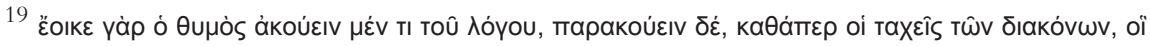

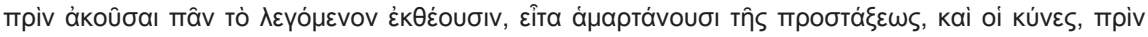

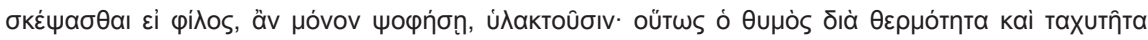

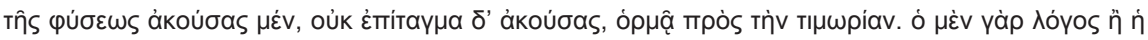

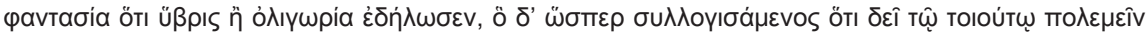

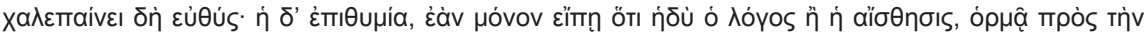

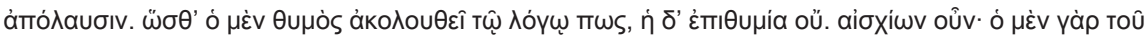

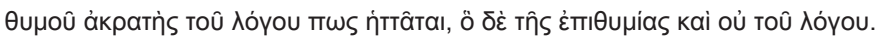

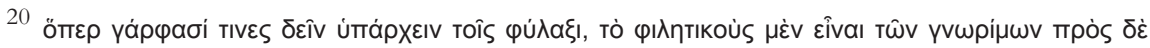

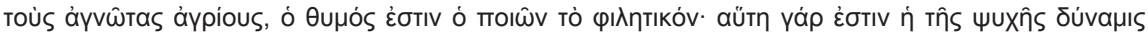

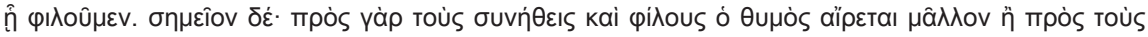

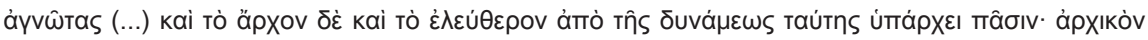

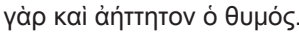

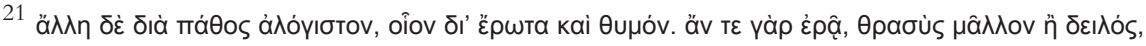

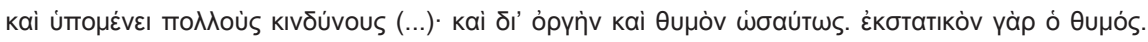

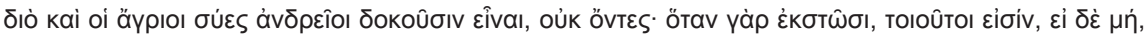

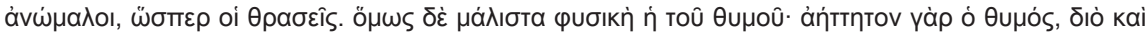

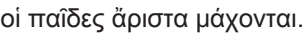

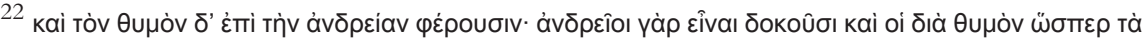

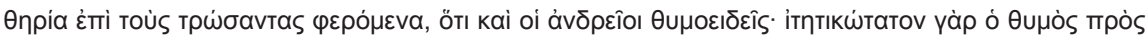

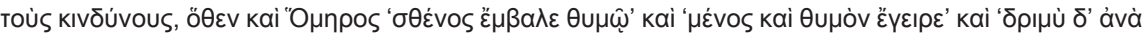

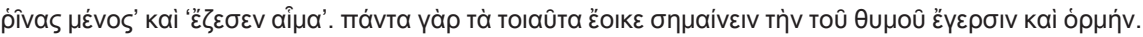

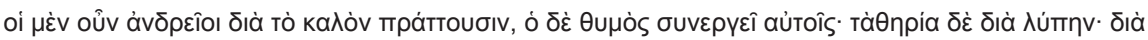

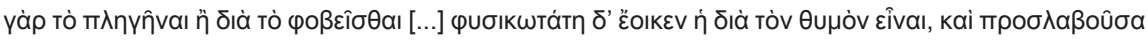

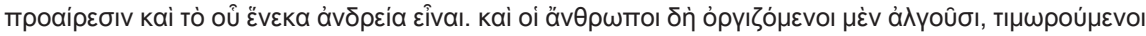

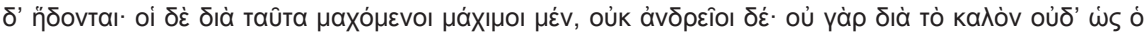

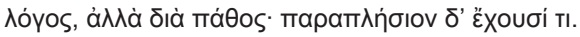

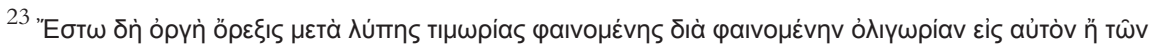

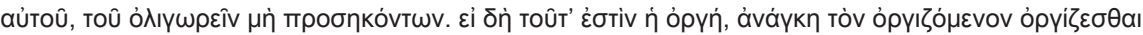

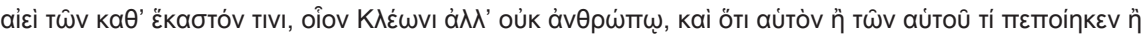

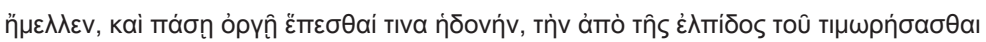

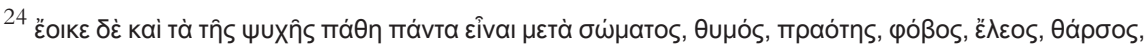

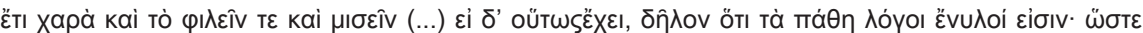

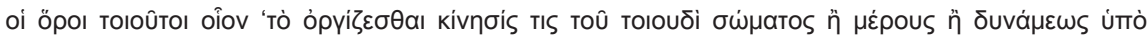

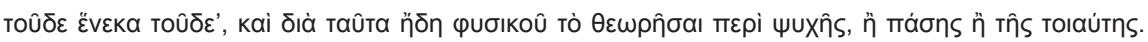

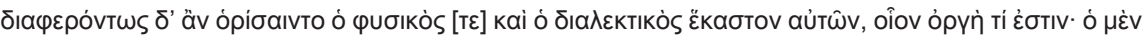

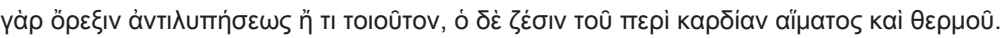

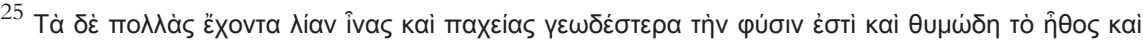

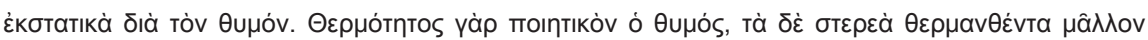

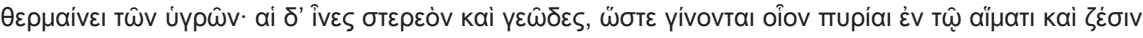




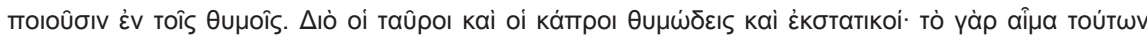

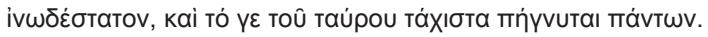

${ }^{26}$ Per le interpretazioni materialistiche del frammento B 85, cf. Burnet 1930 secondo cui il soddisfacimento del desiderio del thumos implica la trasformazione dell'anima secca-fuoco in umidità. Cf. anche Schofield 1983, p. 208 n 2.

27 De iuv., 4, 469 b 14-16: «Se il cuore perde il suo calore, la vita si spegne completamente, poichè tutti gli organi dipendono da questa fonte di calore e l'anima si trova come infiammata (hosper empepureumenês) in questa parte». Cf. a questo proposito Lefevre 1972 e Nuyens 1948.

${ }^{28} \mathrm{O}$ forse anche come un' espressione stilisticamente brillante, capace di migliorare il discorso, come teorizzato in Rhet. III, 10. Una forma di citazione analoga si puo' trovare nella citazione di due frammenti di Empedocle in Meteor. IV, rispettivamente in 4, 381 b 32 ( = 31 B 34 Diels): «... l'umido è per il secco la causa che lo determina e sono l'uno per l'altro come una specie di colla, come Empedocle si esprime nel suo poema Sulla natura: ' facendo colla di farina con dell'acqua'", e in 9, 387 a 32 (= 31 B 82 Diels): " Faccio rientrare in questa classe anche le ossa, i peli e tutte le cose di questo stesso genere: infatti non esiste un nome comune per tutte queste sostanze, tuttavia sono tutte comprese nella stessa classe, per analogia, nel modo in cui si esprime Empedocle: 'identici i peli, le foglie, le piume dense degli uccelli e le scaglie che si formano sulle membra indurite'».

${ }^{29}$ Mansfeld 1992 la individua nell'interpretazione morale «forte » di Aristotele, come conflitto tra desiderio e ragione.

${ }^{30}$ Zaborowski 2003 sottolinea proprio questo aspetto della potenza del thumos come particolarmente interessante per lostudio della dinamica delle forze psichiche dell'antropologia e della psicologia moderne.

\section{Referências bibliográficas}

BOLLACK, J. -WISMANN, H. (1972): Héraclite ou la séparation, Paris.

BURNET, J.(1930): Early Greek Philosophy, London.

CAPIZZI, A. (1979): Eraclito e la sua leggenda, Roma.

COLLI, G. ( 1980): La sapienza greca, v. III: Eraclito, Torino.

CONCHE, M. (1986): Heraclite. Fragments, Paris.

DIANO, C. - SERRA, G. (1980): Eraclito. I frammenti e le testimonianze, Milano.

DIELS, H. (1909): Herakleitos von Ephesos, Berlin.

DIELS, H. - KRANZ, W. (1951-26): Die Fragmente der Vorsokratiker, Berlin. 
FRONTEROTTA, F. (2013): Eraclito, Frammenti, Milano.

INDELLI, G. (1988): Filodemo, L'ira, Napoli.

KAHN, CH. (1979): The Art and Thought of Heraclitus, Cambridge.

KIRK, G.S.- Raven J.E. -Schofield, M. $\left(1983^{2}\right)$ : The Presocratic

Philosophers, Cambridge.

MANSFELD, J. (1992): «Heraclitus fr. B 85 DK», Mnemosyne, 15/1, p. 9-18.

LEFÈVRE, Ch. (1972): Sur l'évolution d'Aristote en psychologie, Louvain, 1972.

MARCOVICH, M. (1978): Eraclito. Frammenti, Firenze, 1978 (ed. maior, Merida, 1967).

MERCKEN, H.P.F. (1980), : «The Greek commentators on Aristotle's Ethics», in R. Sorabji ed., Aristotle transformed, London, 1980, p.407-422.

MOURAVIEV, S. N. (1991): Héraclite d'Ephèse, «Les Muses» ou «De la nature», Moscou-Paris, 1991.

MORAUX, P. (1973):Der Aristotelismus dei den Griechen, Berlin and NY, 1973.

MOURAVIEV, S.N. (2006): Heraclitea, Sankt Augustin.

NUYENS, F. (1948) L'évolution de la psychologie d'Aristote, Louvain.

RAMNOUX, Cl. (1968): Héraclite ou l'homme entre les choses et les mots, Paris.

ROBINSON, T. M. (1987): Heraclitus. Fragments, Toronto.

TSOUNA,V. (2008): «Philodème sur la colère», Philosophie antique 8, p. 217-58.

ZABOROWSKI, R. (2003): «Sur le fragment DK 22B85 d'Héraclite d'Ephèse», Organon 32, p. 9-30.

ZELLER, E- Mondolfo, R. (19682): La Filosofia dei Greci nel suo sviluppo storico, Firenze. 